\title{
Spinopelvic sagittal balance: what does the radiologist need to know?
}

\author{
Equilíbrio sagital espinopélvico: o que o radiologista precisa saber?
}

\section{Leonor Garbin Savarese ${ }^{1, a}$, Rafael Menezes-Reis ${ }^{1, b}$, Gustavo Perazzoli Bonugli ${ }^{1, c}$, Carlos Fernando Pereira da Silva Herrero ${ }^{1, d}$, Helton Luiz Aparecido Defino ${ }^{1, e}$, Marcello Henrique Nogueira-Barbosa ${ }^{1, f}$}

1. Hospital das Clínicas da Faculdade de Medicina de Ribeirão Preto da Universidade de São Paulo (HCFMRP-USP), Ribeirão Preto, SP, Brazil.

Correspondence: Dra. Leonor Garbin Savarese. Hospital das Clínicas da Faculdade de Medicina de Ribeirão Preto da Universidade de São

Paulo, Divisão de Radiologia. Avenida Bandeirantes, 3900, Vila Monte Alegre. Ribeirão Preto, SP, Brazil, 14048-900. Email: leonorgs@usp.br / leonorsavarese@hotmail.com.

a. https://orcid.org/0000-0002-1372-9162; b. https://orcid.org/0000-0002-8496-5939; c. https://orcid.org/0000-0003-2740-3371; d. https://orcid.org/0000-0002-3387-4797; e. https://orcid.org/0000-0003-4274-0130; f. https://orcid.org/0000-0002-7436-5315.

Received 27 March 2019. Accepted after revision 12 July 2019.

How to cite this article:

Savarese LG, Menezes-Reis R, Bonugli GP, Herrero CFPS, Defino HLA, Nogueira-Barbosa MH. Spinopelvic sagittal balance: what does the radiologist need to know? Radiol Bras. 2020 Mai/Jun;53(3):175-184.

Abstract Sagittal balance describes the optimal alignment of the spine in the sagittal plane, resulting from the interaction between the spine and lower limbs, via the pelvis. Understanding sagittal balance has gained importance, especially in the last decade, because sagittal imbalance correlates directly with disability and pain. Diseases that alter that balance cause sagittal malalignment and may trigger compensatory mechanisms. Certain radiographic parameters have been shown to be clinically relevant and to correlate with clinical scores in the evaluation of spinopelvic alignment. This article aims to provide a comprehensive review of the literature on the spinopelvic parameters that are most relevant in clinical practice, as well as to describe compensatory mechanisms of the pelvis and lower limbs.

Keywords: Spine; Pelvis; Radiography; Lordosis/diagnostic imaging; Kyphosis/diagnostic imaging; Spinal curvatures/diagnostic imaging; Postural balance.

Resumo O equilíbrio sagital descreve o alinhamento ideal da coluna vertebral no plano sagital, sendo resultante da interação entre a coluna e os membros inferiores, por meio da bacia. O entendimento do equilíbrio sagital ganhou importância principalmente na última década, uma vez que o desequilíbrio sagital se correlaciona diretamente com incapacidade e dor. Doenças que alterem este equilíbrio podem causar mal alinhamento sagital e desencadear mecanismos compensatórios, os quais tentam restaurar a postura ereta. A literatura relata parâmetros radiográficos clinicamente relevantes, que possuem relação estabelecida com escores clínicos, para avaliar o alinhamento espinopélvico. Este artigo tem como objetivo fornecer uma ampla revisão da literatura sobre os parâmetros espinopélvicos mais relevantes na prática clínica, e descrever os mecanismos compensatórios da pelve e membros inferiores.

Unitermos: Coluna; Pelve; Radiografia; Cifose/diagnóstico por imagem; Lordose/diagnóstico por imagem; Curvaturas da coluna vertebral/diagnóstico por imagem; Equilíbrio postural.

\section{INTRODUCTION}

The human spine presents high biomechanical complexity, allowing bipedalism and resulting in the development of S-shaped curvatures of the spine ${ }^{(1)}$. The relationship between the pelvis and the spine is a direct effect of bipedalism. Adopting a vertical posture resulted in the expansion and the verticalization of the pelvis, which has led to the emergence of sagittal curves in the spine-including cervical lordosis, thoracic kyphosis, and lumbar lordosis-as well as to modifications in the tissues that stabilize the spine ${ }^{(2)}$.

Spinal deformity in the sagittal plane has been considered one of the main causes of disability, with a significant impact on health, and reputed by some authors as equivalent to diseases such as cancer, diabetes, and heart disease $^{(3-7)}$. As the western population has aged, adult spine deformity has become more prevalent, with estimated rates of up to $60 \%$ in the elderly population of the United States ${ }^{(8)}$. The understanding of sagittal balance has gained importance in the last decade, with evidence that an imbalance is directly correlated with disability and pain $^{(3)}$.

Introduced in the mid-1980s, the concept of sagittal balance has been widely used in the evaluation and management of disorders of the spine, with a growing interest in the study of spinopelvic parameters over the last three decades $^{(3-9)}$. Continuing the work of During et al. ${ }^{(10)}$ and Duval-Beaupère et al. ${ }^{(11)}$, several authors have emphasized the importance of sagittal balance in diseases of the spine $^{(12,13)}$. Roussouly et al. ${ }^{(14)}$ played a fundamental role 
in promoting the concept, creating a classification of the asymptomatic population based on spinopelvic parameters. Other studies involving asymptomatic patients showed correlations between spinopelvic parameters and sagittal curvatures of the spine. One study, conducted by Glassman et al. ${ }^{(3)}$, became a classic reference that established the sagittal vertical axis as the primary measure of sagittal deformity.

The present article aims to provide a comprehensive review of the literature on the spinopelvic parameters that are most relevant in clinical practice, as well as to describe the compensatory mechanisms of the pelvis and lower limbs.

\section{THE EXAMINATION TECHNIQUE}

For the radiographic evaluation of the sagittal plane, it is necessary to acquire a panoramic lateral view of the spine that enables evaluation all the way from the craniovertebral junction to the femoral heads, which would need to be included within the field of vision and with visible contours. There are different ways of positioning the patient for a panoramic spine radiography. Based on the study of Marks et al. ${ }^{(15)}$, the patient should be standing, with the upper limbs resting on a support, the shoulders at $30^{\circ}$ forward flexion, and the elbows slightly flexed (Figure 1). That position is most comparable to the functional standing position, in which both arms hang at the sides, and does not significantly alter the sagittal alignment ${ }^{(15)}$.

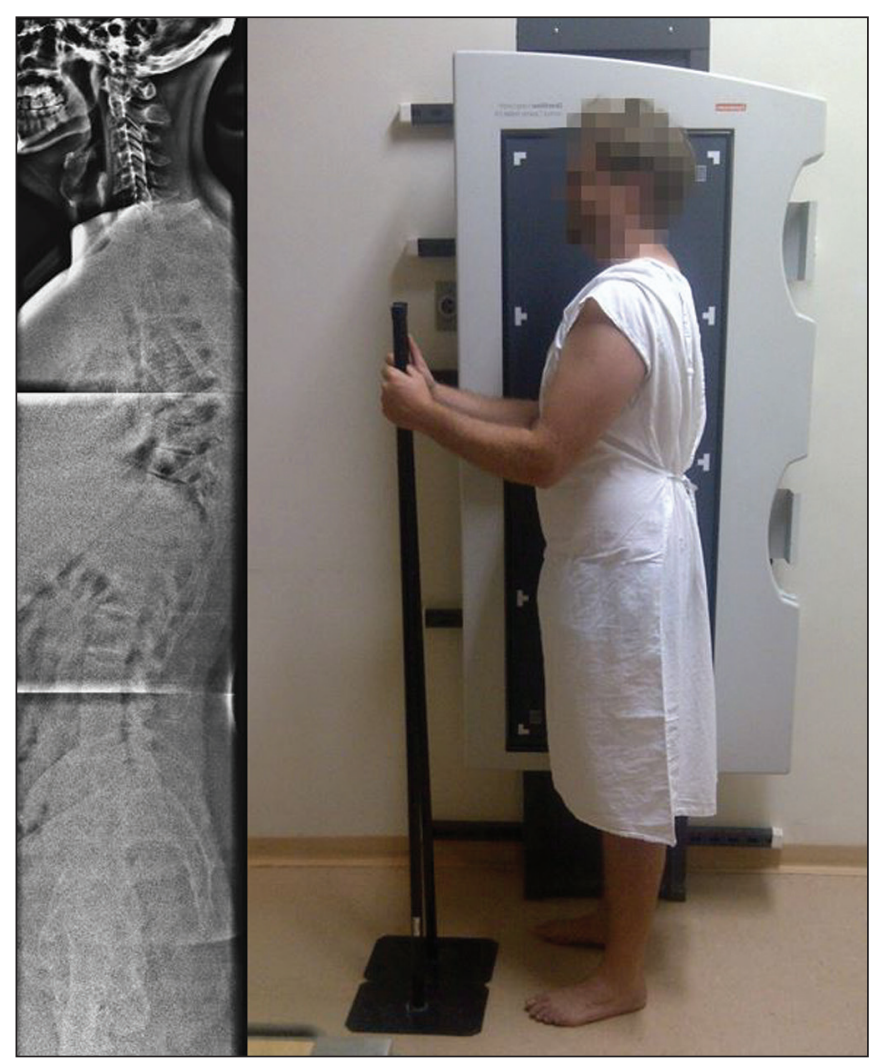

Figure 1. Panoramic $X$-ray acquired in the sagittal plane (on the left) and patient positioning for its acquisition (on the right).

\section{SPINOPELVIC PARAMETERS}

\section{Pelvic parameters}

The most commonly used pelvic parameters are the pelvic tilt, the sacral slope, and the pelvic incidence.

\section{Pelvic tilt}

The pelvic tilt is the angle formed between the vertical and a line connecting the midpoint of the femoral heads to the midpoint of the upper endplate of S1 (Figure $2)$. In simple terms, this angle describes the rotational orientation of the pelvis around the femoral heads. It varies between $5^{\circ}$ and $30^{\circ}$, with an average of $12^{\circ(5,16,17)}$. In most cases, the two femoral heads do not overlap perfectly in the lateral view. In such cases, we should use the geometric center of the femoral heads, which is the midpoint of the line that connects the geometric centers of the femoral heads. Lafage et al. ${ }^{(6)}$ demonstrated that a greater pelvic tilt correlates with worse quality of life and poorer health status.

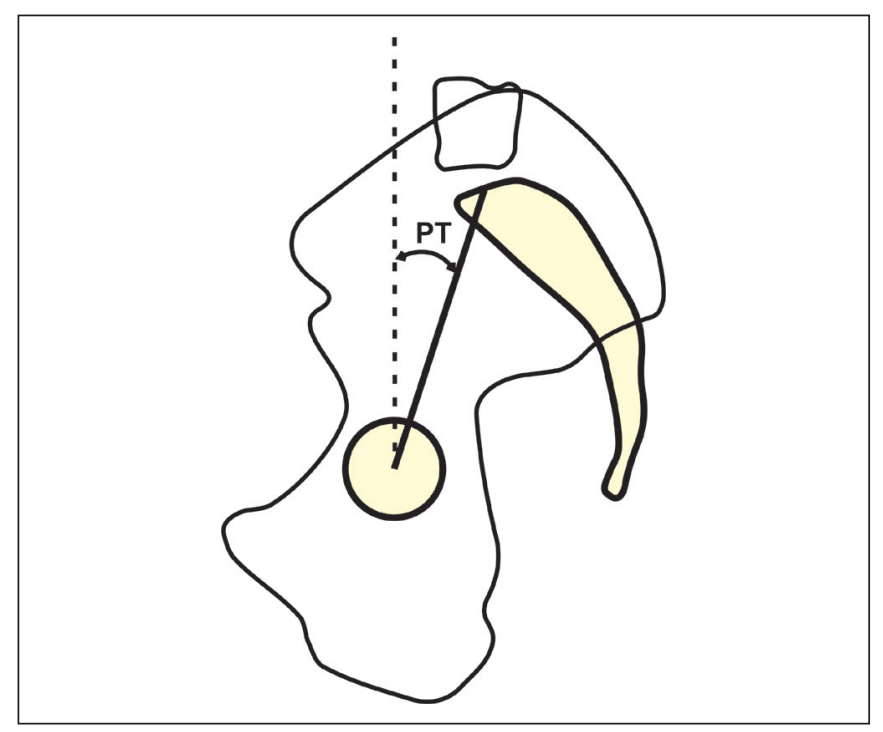

Figure 2. Schematic representation of the pelvic tilt (PT), which is the angle formed by a vertical line originating from the midpoint of the femoral head and a line running from the midpoint of the femoral head to the midpoint of the upper endplate of S1.

\section{Sacral slope}

The sacral slope is the angle formed between the horizontal and the upper endplate of S1 (Figure 3). It varies between $20^{\circ}$ and $65^{\circ}$, with an average of $40^{\circ(16)}$, and has a direct correlation with lumbar lordosis.

\section{Pelvic incidence}

The pelvic incidence is the angle between a perpendicular line to the upper endplate of S1 and the line connecting the midpoint of that endplate with the midpoint of the femoral rotation (Figure 4).

The pelvic incidence is a morphological parameter that is independent of the spatial orientation of the pelvis 


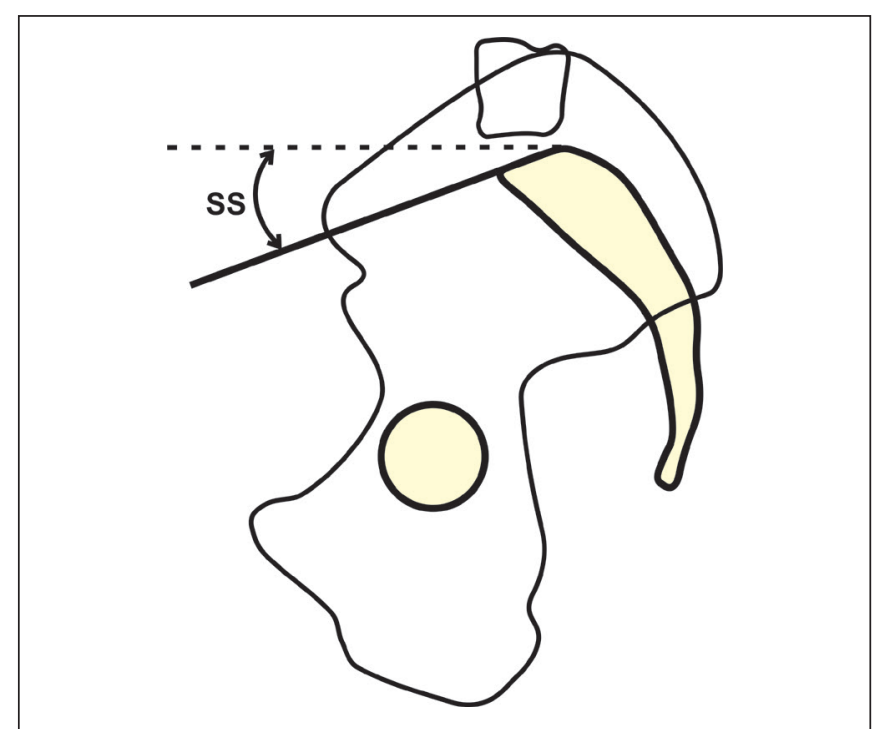

Figure 3. Schematic representation of the sacral slope (SS) angle, which is the angle between the upper endplate of S1 and a horizontal line.

and is considered specific for each individual ${ }^{(5)}$. The pelvic incidence value grows progressively during adolescence and becomes constant in adulthood. It is directly related to the value of the lumbar lordosis and ranges from $34^{\circ}$ to $84^{\circ}$, with an average of $52^{\circ(5,16,17)}$. There is a geometric relationship between those parameters: pelvic incidence $=$ pelvic tilt + sacral slope. It is important to understand that the pelvic incidence is a descriptive parameter of pelvic morphology and not of its orientation, therefore, its angular value is not affected by postural changes. In contrast, the pelvic tilt and sacral slope vary and are useful for characterizing the spatial orientation of the pelvis. The pelvic incidence is related to the ability that each individual has

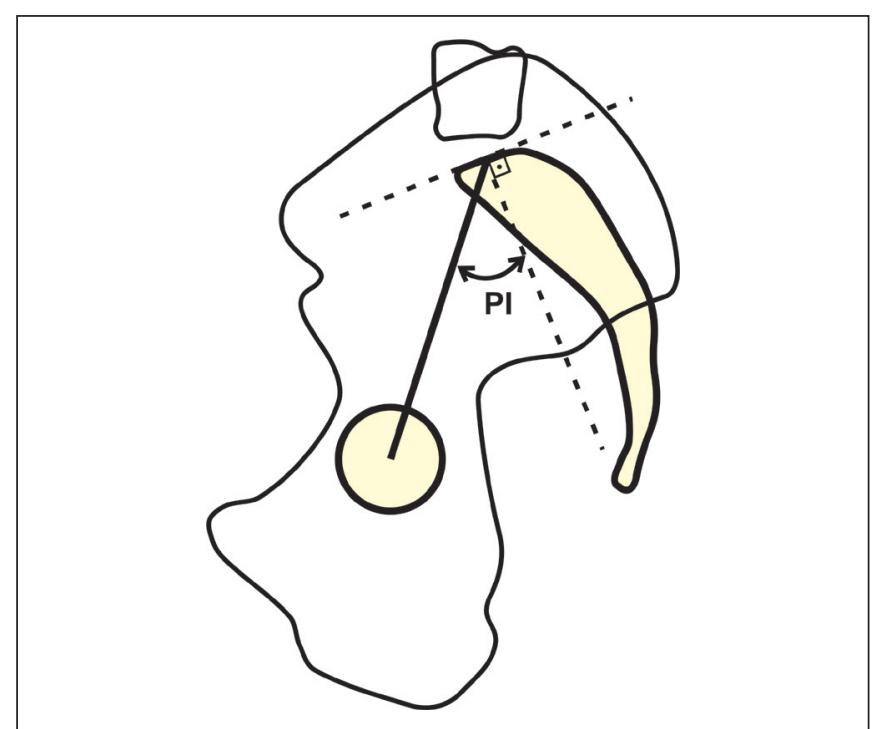

Figure 4. Schematic representation of the pelvic incidence (PI), which is the angle between a line perpendicular to the upper endplate of S1 and a line connecting the midpoint of that endplate with the midpoint of the femoral head.

to compensate for sagittal imbalance. A high pelvic incidence accompanied by a high sacral slope and a low pelvic tilt indicates a greater capacity for spinopelvic compensation. In contrast, a low pelvic incidence indicates less capacity for spinopelvic compensation.

The rotation of the pelvis (a change in the pelvic tilt) causes combined movements of rotation and shifting of the S1 vertebra. These dislocations result in changes in the sacral slope and modify the relative position of the lumbosacral junction in relation to the hips (Figure 5). Therefore, increasing the pelvic tilt (extending the hips) is a posterior shift that will decrease the sacral slope through verticalization of the S1. Conversely, decreasing

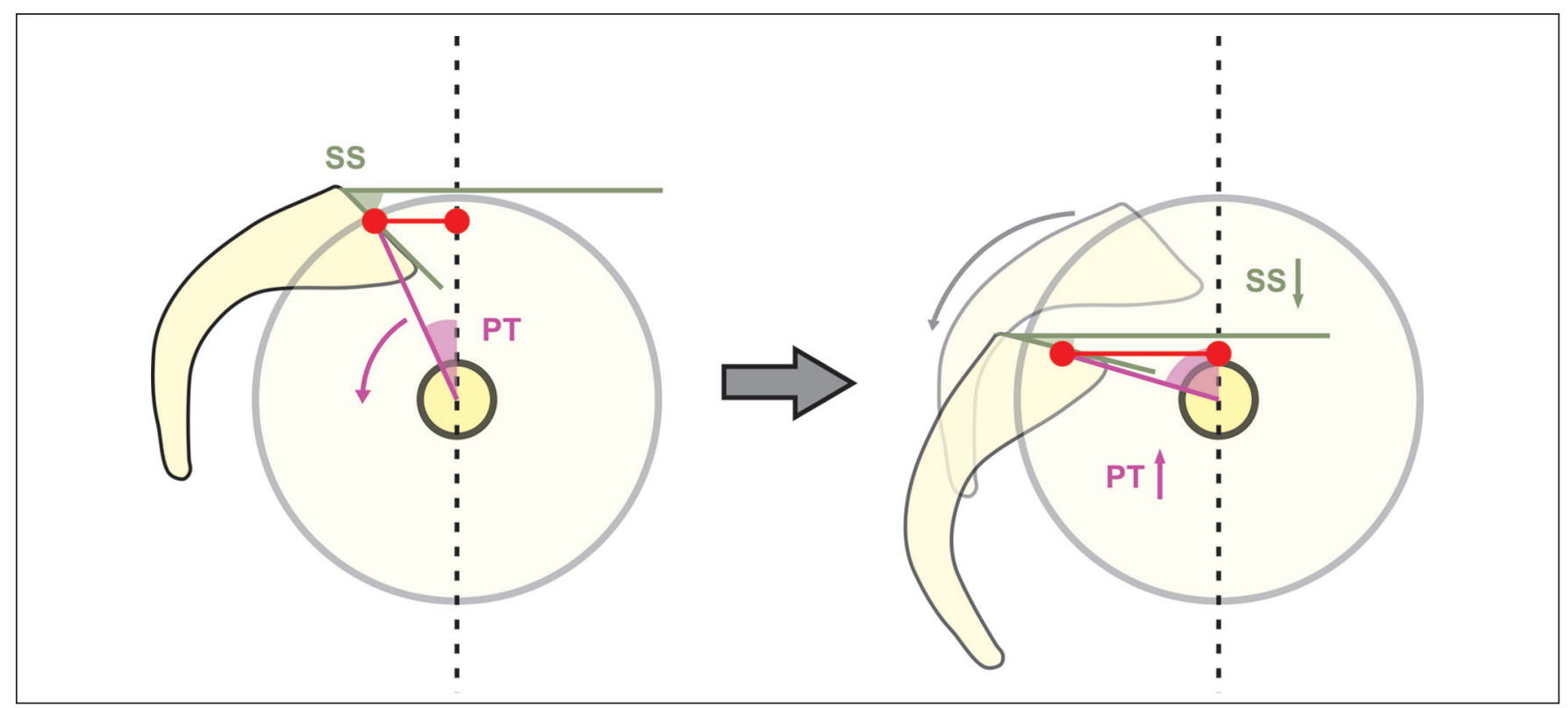

Figure 5. Schematic representation of pelvic retroversion. By bringing the upper endplate of S1 closer to the axis of the femoral heads and increasing the sacrofemoral distance, this mechanism compensates for the anterior shift in the center of gravity. Adapted from Barrey et al. ${ }^{(9)}$. SS, sacral slope; PT, pelvic tilt. 
the pelvic tilt (flexing the hips) results in an anterior shift that will increase the sacral slope through horizontalization of the $S 1^{(17-20)}$.

\section{Vertebral parameters}

The most commonly used vertebral parameters are thoracic kyphosis, lumbar lordosis, the sagittal vertical axis, the difference between the pelvic incidence and lumbar lordosis (PI-LL), the T1 pelvic angle, global tilt, the spinopelvic angle, and the spinosacral angle.

\section{Thoracic kyphosis}

The identification of thoracic kyphosis is based on the measurement of the Cobb angle between the lower endplate of T12 and the upper endplate of $\mathrm{T} 1^{(21)}$. However, the position of the shoulder on an X-ray can overlap the image and hinder the localization of T1. To avoid this problem, some authors suggest using the upper endplate of T4 to measure thoracic kyphosis ${ }^{(22)}$. As depicted in Figure $6 \mathrm{~A}$, the Cobb angle between $\mathrm{T} 4$ and $\mathrm{T} 12$ ranges from $20^{\circ}$ to $50^{\circ}$ in normal individuals ${ }^{(23)}$.

\section{Lumbar lordosis}

The identification of lumbar lordosis is based on the measurement of the Cobb angle between the upper endplate of $\mathrm{S} 1$ and the upper endplate of $\mathrm{Ll}^{(6,24,25)}$, as shown in Figure 6B. It normally ranges between $30^{\circ}$ and $79^{\circ}(18,19,26)$.

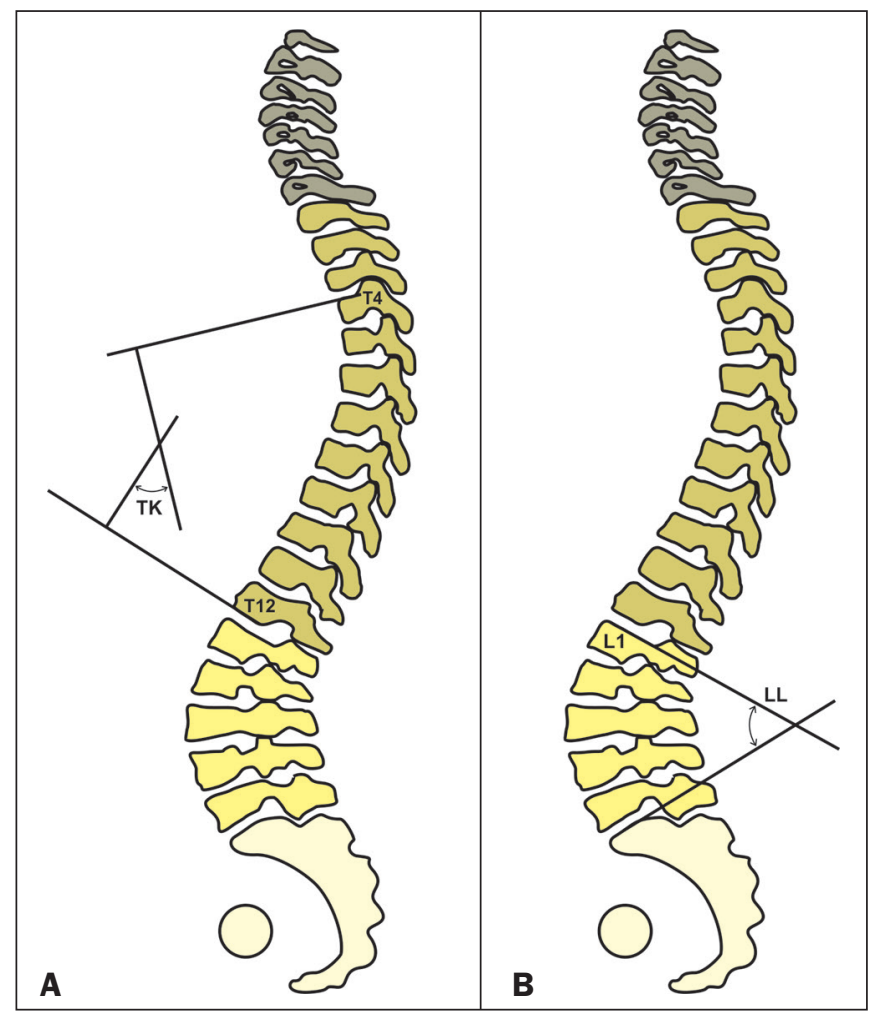

Figure 6. A: Schematic representation of the thoracic kyphosis (TK) angle, which is the measurement of the Cobb angle between the lower endplate of T12 and the upper endplate of T4. B: Schematic representation of the lumbar lordosis (LL) angle, which is the measurement of the Cobb angle between the upper endplate of S1 and the upper endplate of L1.
Two recent studies have used statistical calculations to predict the ideal lumbar lordosis using formulas based on pelvic incidence. With a multilinear regression analysis, Legaye et al. ${ }^{(27)}$ proposed a formula for the prediction of the ideal lumbar lordosis: lumbar lordosis $=-[$ (pelvic incidence $\times 0.5481+12.7) \times 1.087+21.61]$. Subsequently, Schwab et al. ${ }^{(22)}$ proposed a simpler approach, and estimated the ideal lumbar lordosis based on an asymptomatic adult population (75 individuals; mean age of $48 \pm 18$ years $)$ : lumbar lordosis $=$ pelvic incidence $+9^{\circ}( \pm 9)$.

\section{Sagittal vertical axis}

The sagittal vertical axis is the measurement of the horizontal distance between the $\mathrm{C} 7$ plumbline and the vertical line passing through the upper posterior edge of S1 (Figure 7). This serves to document the location of the head in relation to the center of gravity ( $\mathrm{C} 7$ deviation in relation to the sacral promontory). Jackson et al. ${ }^{(18)}$ reported values in asymptomatic adults with a mean sagittal vertical axis deviation of $0.5 \pm 2.5 \mathrm{~cm}$. Glassman et al. ${ }^{(3)}$ showed that, among 352 patients with positive sagittal alignment, a high sagittal vertical axis correlated with pain and worse scores for health and quality of life.

\section{PI-LL}

The PI-LL is often used as a descriptive parameter for the spine alignment ${ }^{(6,18)}$. Schwab et al. ${ }^{(28)}$ found that

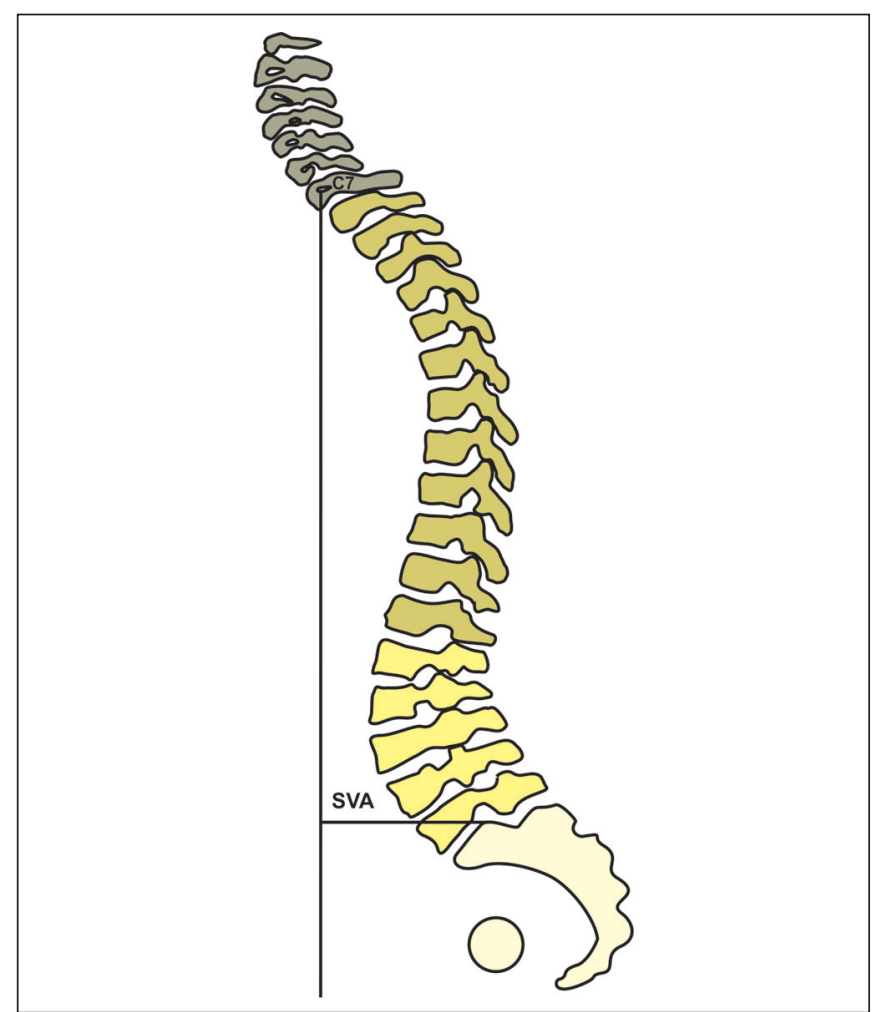

Figure 7. Schematic representation of the sagittal vertical axis (SVA), which is the horizontal distance between the $\mathrm{C} 7$ plumbline and the posterior edge of the upper endplate of S1. 
the PI-LL correlated significantly with the pelvic tilt $(\mathrm{r}=$ $0.844 ; p<0.001)$ and the sagittal vertical axis $(\mathrm{r}=0.685 ; p$ $<0.001)$. It has been suggested that a PI-LL below $10^{\circ}$ indicates a malalignment. Because the lumbar lordosis value must adapt to the pelvic morphology (evaluated by the pelvic incidence), a lack of correspondence between the two values would represent a condition in which the patient could not find a spinopelvic organization in accordance with their pelvic anatomy. The PI-LL showed a correlation with questionnaires related to health and quality of life, simultaneously proving to be a valuable tool for the intraoperative planning of correction of flat back syndrome ${ }^{(4,6,7,29)}$, being used as the basis for determining the target correction in surgical treatment of sagittal malalignment.

\section{T1 pelvic angle and global tilt}

Recently, the T1 pelvic angle and the global tilt were proposed as new spinopelvic parameters that represent both spinal inclination and pelvic retroversion. These parameters do not change with postural compensation ${ }^{(4,30)}$.

The T1 pelvic angle is the angle between the line running from the geometric midpoint of the femoral heads to the midpoint of the vertebral body of $\mathrm{T} 1$ and that running from the geometric midpoint of the femoral heads to the midpoint of the upper endplate of S1 (Figure 8). It corresponds to the sum of the T1 spinopelvic inclination and the pelvic tilt.

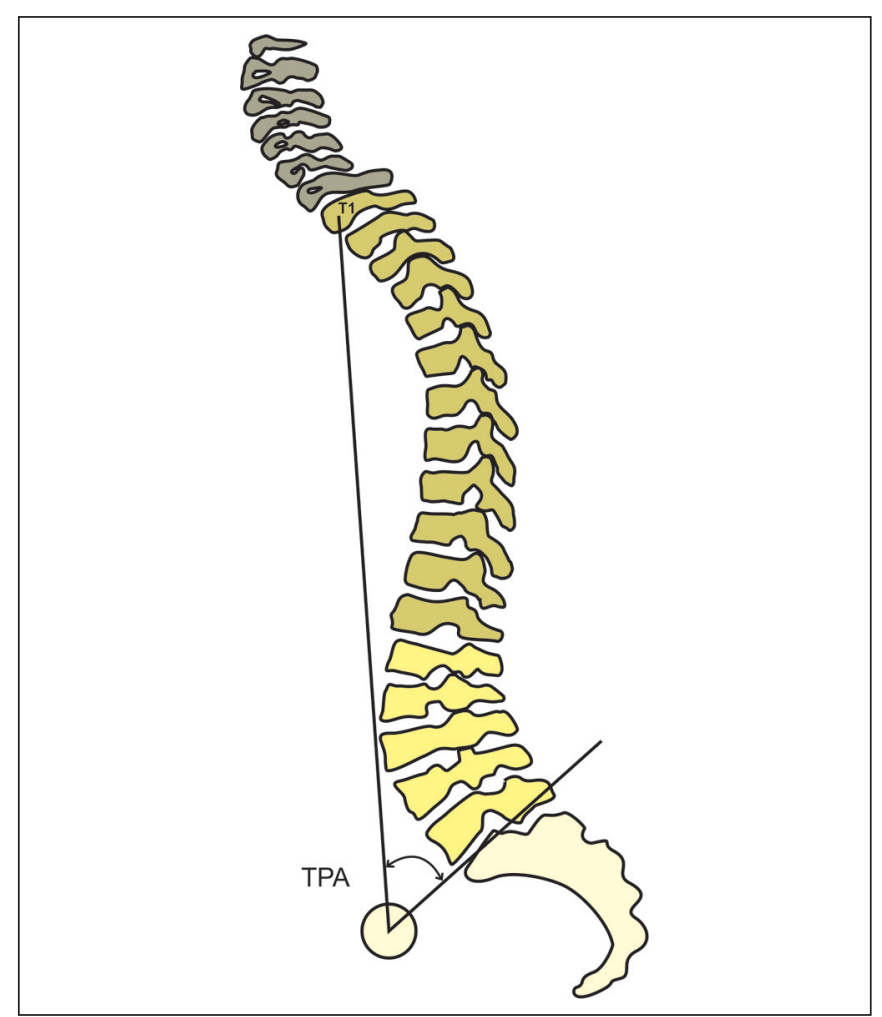

Figure 8. Schematic representation of the T1 pelvic angle (TPA), which is the angle between the line running from the midpoint of the vertebral body of T1 to the geometric midpoint of the femoral heads and the line running from the geometric midpoint of the femoral heads to the midpoint of the upper endplate of S1.
In the study conducted by Protopsaltis et al. ${ }^{(4)}$, the T1 pelvic angle was found to correlate with the sagittal vertical axis $(r=0.837)$, the PI-LL $(r=0.889)$, and the pelvic tilt $(r=0.933)$, as well as with questionnaires related to the health and quality of life of patients with adult spinal deformity. The authors suggested that it is a useful tool in the preoperative planning for such patients, the target T1 pelvic angle being $<14^{\circ}$.

The global tilt is the angle between the line running from the midpoint of the upper endplate of $\mathrm{S} 1$ to the midpoint of the vertebral body of $\mathrm{C} 7$ and that running from the geometric midpoint of the femoral heads to the midpoint of the upper endplate of S1 (Figure 9).

The T1 pelvic angle and the global tilt combine the trunk anteversion and pelvic retroversion as a parameter to evaluate the overall spinal deformity. Recently, Banno et al. ${ }^{(31)}$ described reference values for the global tilt in elderly individuals. In that study, the global tilt seemed to be related to age - the mean global tilt being $15.4 \pm 8.7^{\circ}$ for the patients between 50 and 59 years of age and 30.8 $\pm 14.8^{\circ}$ for the patients $\geq 80$ years of age-and genderthe mean global tilt being $26.0^{\circ}$ for women and $18.8^{\circ}$ for men-showing a high correlation with the pelvic tilt ( $\mathrm{r}$ $=0.914)$ and the sagittal vertical axis $(r=0.751)$. The authors proposed a cutoff global tilt value of $33.7^{\circ}$ for an Oswestry Disability Index of $>40$. These results underscore the fact that the interpretation of the global tilt

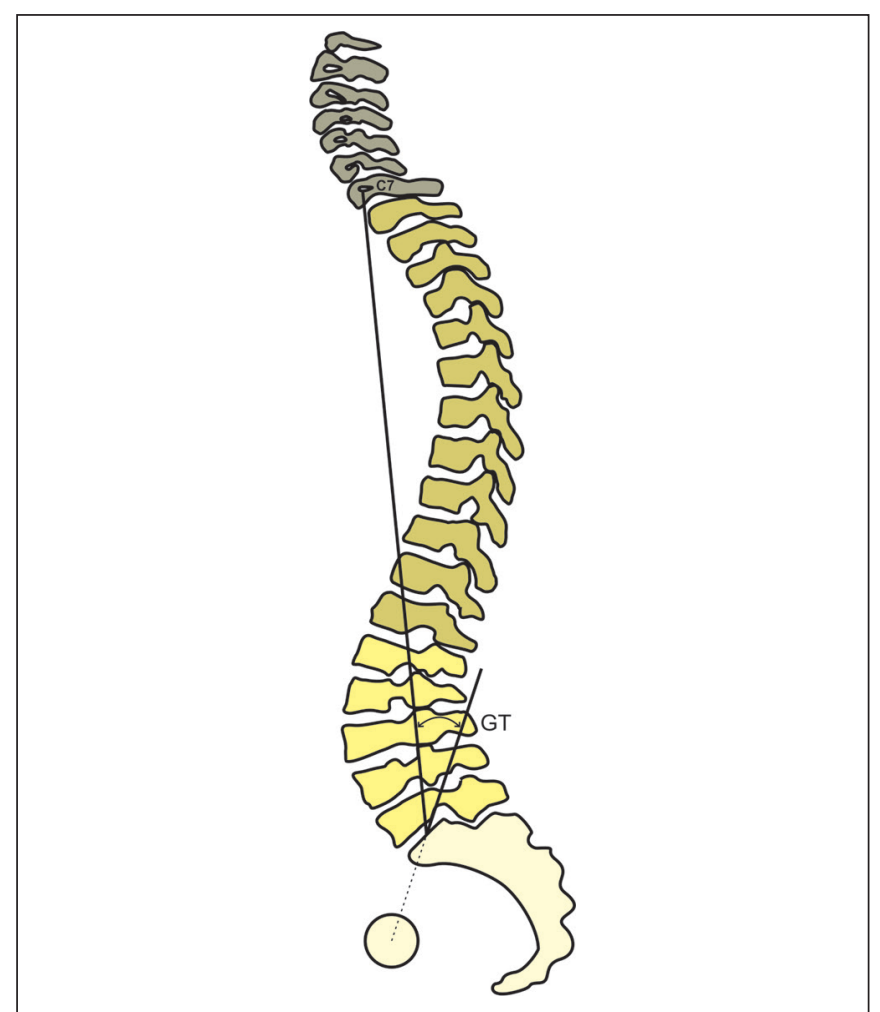

Figure 9. Schematic representation of the global tilt (GT), which is the angle between the line running from the midpoint of the vertebral body of $\mathrm{C} 7$ to the midpoint of the upper endplate of $\mathrm{S} 1$ and the line running from the midpoint of the upper endplate of S1 to the geometric midpoint of the femoral heads. 
values, like that of other sagittal parameters, should be made according to the general context of patients with deformities of the spine.

\section{Spinopelvic angle}

The spinopelvic angle is the angle between the line running from the midpoint of $\mathrm{C} 7$ to the midpoint of the upper endplate of $\mathrm{S} 1$ and that running from the midpoint of the upper endplate of $\mathrm{S} 1$ to the geometric midpoint of the femoral heads (Figure 10). This angle evaluates the overall spinopelvic alignment, taking into consideration the retroversion of the pelvis and the anteversion of the trunk.

\section{Spinosacral angle}

As depicted in Figure 11, the spinosacral angle is the angle between the line running from the midpoint of $\mathrm{C} 7$ to the midpoint of the upper endplate of $\mathrm{S} 1$ and the surface of that endplate; it was conceived as a means of quantifying the overall kyphosis of the spine as a whole. The average value of the spinosacral angle in asymptomatic individuals is $130.4 \pm 8.1^{\circ(32)}$. In a study involving a healthy population ${ }^{(19)}$, the spinosacral angle was found to correlate strongly with the sacral slope $(\mathrm{r}=0.914 ; p<0.001)$ and the lumbar lordosis $(\mathrm{r}=0.889 ; p<0.001)$. Other studies have shown that the spinosacral angle is lower in the presence of

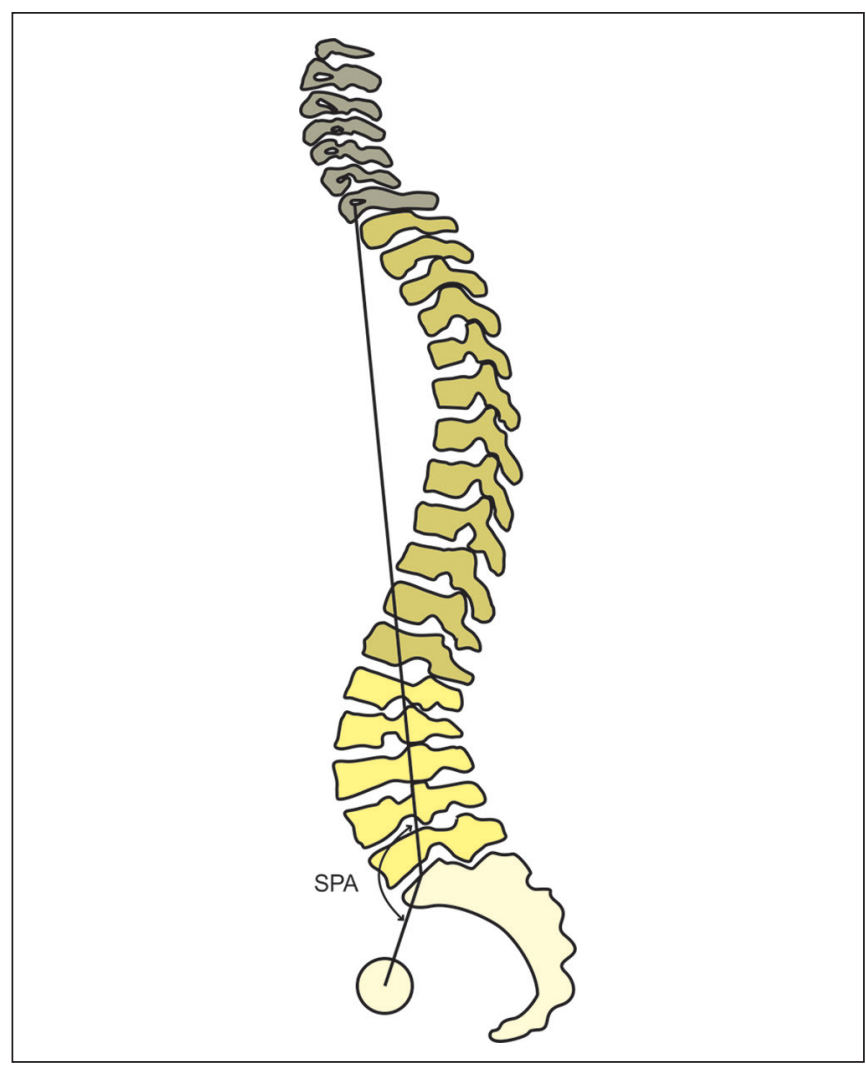

Figure 10. Schematic representation of spinopelvic angle (SPA), which is the angle between the line running from the midpoint of $\mathrm{C} 7$ to the midpoint of the upper endplate of $\mathrm{S} 1$ and the line running from the midpoint of the upper endplate of S1 to the geometric midpoint of the femoral heads. diseases such as degenerative kyphosis ${ }^{(33)}$, rheumatoid arthritis ${ }^{(34)}$, and discogenic lumbar pain ${ }^{(35)}$. In addition, the spinosacral angle is associated with the presence of pain and functional alterations ${ }^{(34)}$.

\section{Relationships between spinopelvic parameters and the Roussouly classification}

The recognition of the relationship between pelvic morphology, represented by the pelvic incidence, and the lumbar lordosis improved understanding of malalignment of the spine in the sagittal plane $e^{(5)}$. The pelvic incidence value is an individual anatomical characteristic and corresponds to the "thickness" of the pelvis. More than two decades ago, Legaye et al. ${ }^{(5)}$ and Duval-Beaupère et al. ${ }^{(11)}$ postulated that a high pelvic incidence is associated with a high sacral slope and pronounced lumbar lordosis, and that a low pelvic incidence is associated with a lower sacral slope and subtle lumbar lordosis, leading to the basic concept of an "economic standing position"(36).

Based on the concept that the morphology and spatial orientation of the pelvis determine the organization of the spine and its curvatures, Roussouly et al. ${ }^{(14)}$ created a classification in the asymptomatic population based on their observation of a strong correlation between the lumbar lordosis and the sacral slope. After observing that there are characteristic sagittal profiles that vary depending on the

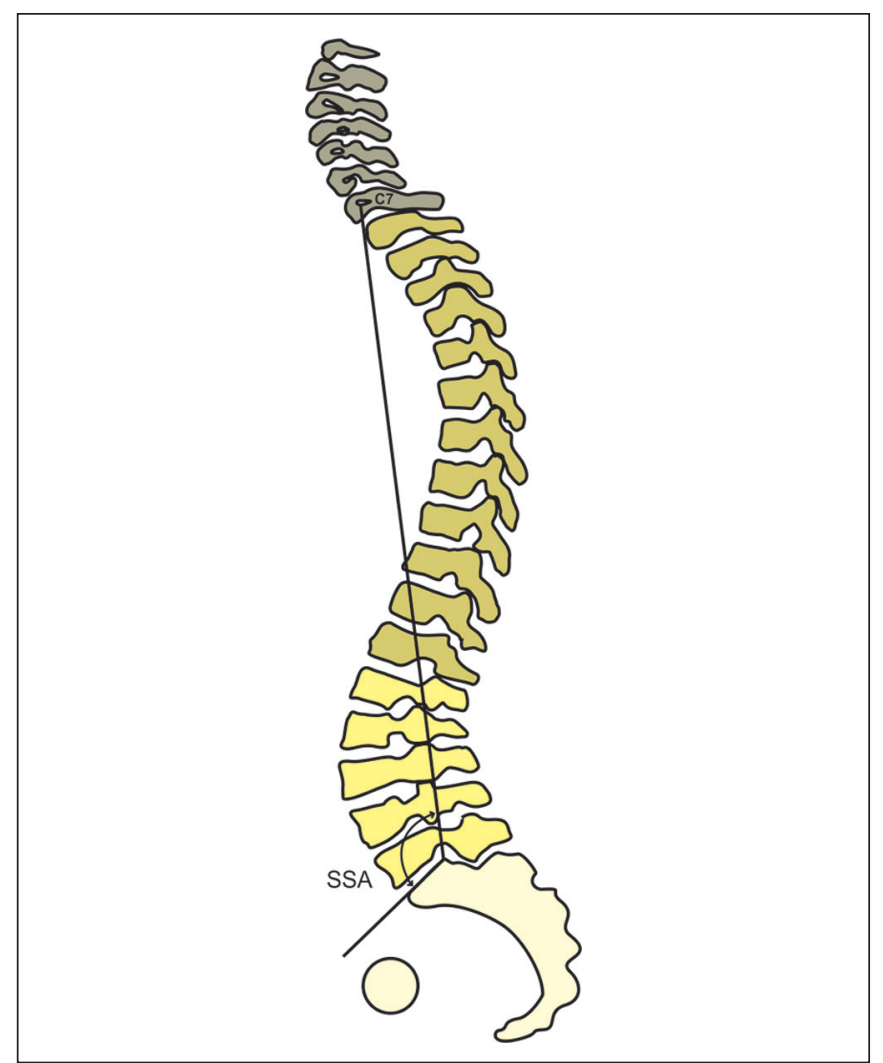

Figure 11. Schematic representation of spinosacral angle (SSA), which is the angle formed by the line that runs from the midpoint of $\mathrm{C} 7$ to the midpoint of the upper endplate of S1 and the surface of that same endplate. 
spatial orientation of the pelvis, the authors described four patterns of sagittal alignment variation, as depicted in Figure 12 . Roussouly type 1 is characterized by an sacral slope value of less than $35^{\circ}$ and a low pelvic incidence. The lower arch of the lordosis is minimal, with a short lumbar lordosis and a long thoracolumbar kyphosis. Type 2 is characterized by an sacral slope value of less than $35^{\circ}$ and a low pelvic incidence, the lower arch of the lordosis being relatively flat. The entire spine is relatively hypolordotic and hypokyphotic. Type 3 is characterized by an sacral slope value between $35^{\circ}$ and $45^{\circ}$, greater prominence of the lower arch of the lordosis, and better balance of the spine, the thoracic kyphosis and lumbar lordosis being in harmony. Type 4 is characterized by an sacral slope value greater than $45^{\circ}$, accompanied by a high pelvic incidence and prominence of the lower arch of the lordosis.

\section{Compensatory mechanisms}

There is no single pattern of sagittal balance in the normal population ${ }^{(20)}$. It is essential to have a very high congruence between the pelvis and the spine to achieve an economic posture with the center of gravity in a given physiological position ${ }^{(20,37)}$. The ultimate goal of spinopelvic balance is to keep the center of gravity near the center of the hips ${ }^{(19)}$. The interaction between the spine and the pelvis is an important key point in the analysis of spinal deformities. This interaction is further modulated by the compensatory mechanisms to maintain a permanent alignment of the horizontal position and horizontal gaze ${ }^{(36,38,39)}$. The sagittal imbalance is basically triggered by a maladjustment between the pelvic incidence and lumbar lordosis, due to disc degeneration, trauma, or iatrogenic fusion. The reduction of the lumbar lordosis results in an anterior flexion of the trunk, characterized by an increase in the sagittal vertical axis, giving rise to a curved posture and a downward tilt of the head, with the consequent inability to see above the horizon. When this imbalance occurs, compensatory mechanisms are activated in order to restore a compensated balance. The compensation begins at the level of the spine with modifications of the curvatures, reduction of the kyphosis, and lumbar hyperextension. If this compensation is insufficient, the patient tilts the pelvis backwards and extends the hips, increasing the value of pelvic tilt and reducing the sagittal vertical axis. In addition, some lumbar segments may present hyperextension or retrolisthesis, thus increasing the risk of stenosis of the vertebral canal.

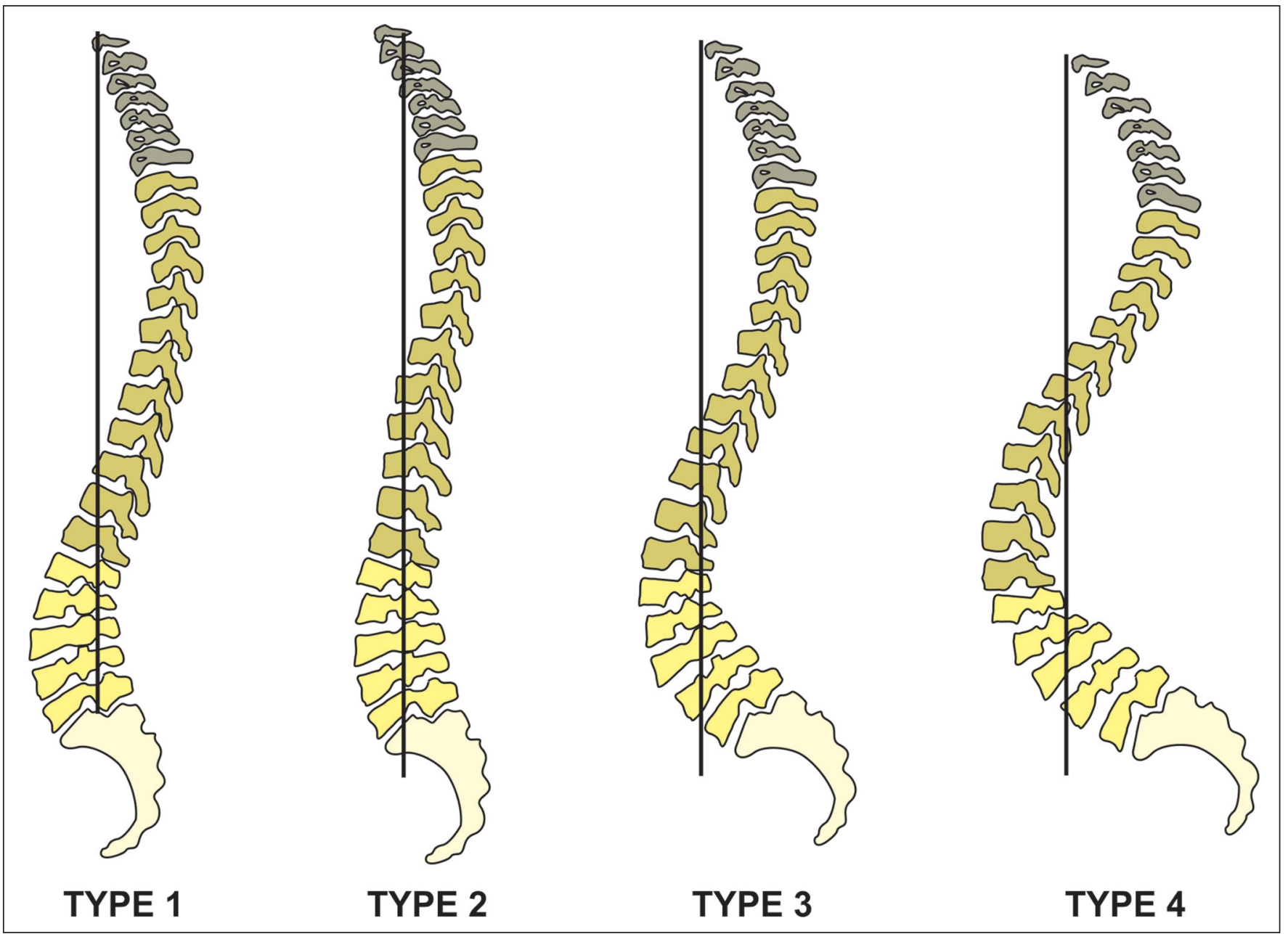

Figure 12. Postural types according to the Roussouly classification. 
Finally, when all compensatory mechanisms have been exhausted, the lower limbs may help restore a horizontal gaze by flexion of the knees ${ }^{(38,40)}$. The flexion of the knees affects the magnitude of the sagittal vertical axis allowing greater pelvic retroversion to retract the head over the hips and ankles, effectively reducing the sagittal vertical $\operatorname{axis}^{(36,38,39)}$.

\section{DISCUSSION}

Studies have confirmed that patients with lumbar degenerative disease are characterized by anterior sagittal imbalance, loss of lumbar lordosis, and increased pelvic tilt $^{(41,42)}$. The sagittal vertical axis and pelvic tilt are spinopelvic parameters that reflect the severity of the adult spine deformity, however, there are some points to be considered. First, the measurement of the sagittal vertical axis can be diminished by postural compensation mechanisms such as pelvic retroversion. Therefore, a high pelvic tilt can "hide" a greater spinal deformity when only the sagittal vertical axis is being considered. The sagittal vertical axis and pelvic tilt are inter-related in the sense that the magnitude of one affects the other. Lafage et al. ${ }^{(6)}$ suggested that the pelvic tilt should be considered in conjunction with the sagittal vertical axis to identify patients with spinal deformity in the sagittal plane without a high sagittal vertical axis due to pelvic compensation. It is worth mentioning that a successful realignment plan should not only restore the spinopelvic relationship but also zero out the compensatory mechanisms, which are energy drains and affect patient quality of life. Therefore, the sagittal vertical axis of the patient should not be considered in isolation when evaluating the sagittal plane. In contrast, the T1 pelvic angle and the global tilt have many advantages for the assessment of global alignment because they consider the retroversion of the pelvis and anteversion of the trunk, as well as because they are not affected by postural or radiographic calibrations ${ }^{(4,38)}$. In addition, the T1 pelvic angle and global tilt both correlate strongly with the sagittal vertical axis, pelvic tilt, and PI-LL ${ }^{(31)}$. Based on these concepts, we suggest the use of the global tilt, spinopelvic angle, and T1 pelvic angle parameters in clinical practice, because they are less prone to postural variation than the sagittal vertical axis.

Roussouly et al. ${ }^{(40)}$ described spinopelvic relationships by means of the spinopelvic angle. The spinopelvic angle is similar in concept to the $\mathrm{T} 1$ pelvic angle and global tilt, because it also assesses the global spinopelvic alignment, taking into consideration the retroversion of the pelvis and the anteversion of the trunk. However, the spinopelvic angle decreases as the deformity increases, and the pathological values of spinopelvic angle that correlate with health-related quality of life have yet to be established. In reality, global tilt is a modified version of the spinopelvic angle described by Roussouly. From a geometric point of view, this angle corresponds to the following formula: spinopelvic angle $=$
180 - global tilt. The spinopelvic angle and global tilt are in fact supplementary angles.

The compensatory mechanisms of the pelvis can be quantified by the pelvic tilt, an angle proposed by DuvalBeaupère et al. $^{(11)}$, which was subsequently correlated with health-related quality of life scores by Lafage et al. ${ }^{(6)}$. Theoretically, individuals with a low pelvic incidence would have a greater anterior acetabular anteversion with greater hip extension and, as a result, less capacity to adapt to sagittal malalignment ${ }^{(43)}$. Lafage et al. ${ }^{(6)}$ demonstrated that, when they categorized patients with deformity into four groups by pelvic tilt (higher and lower) and sagittal vertical axis (higher and lower), the group with the worst score on the Oswestry Disability Index showed higher sagittal vertical axis values and lower pelvic incidence values, suggesting that patients with greater deformities and less ability to compensate with pelvic retroversion would have higher levels of disability.

Roussouly et al. ${ }^{(21)}$ performed a biomechanical analysis of the spinopelvic organization, categorized by types, which has been said to describe the degenerative evolution of the spine. Roussouly et al. ${ }^{(14)}$ suggested that patients with symptomatic disc disease are most commonly classified as types 1 or 2 , while the stenosis of the vertebral canal is usually associated with type 4 , whereas type 3 is rarely seen in patients with disorders of the spine. ChaléatValayer et al. ${ }^{(44)}$ found a higher proportion of patients with chronic lumbar pain whose sacral slope, pelvic incidence, and lumbar lordosis were all lower than the control individuals, suggesting a relationship between this specific pattern (Roussouly type 2) and the presence of lumbar pain. Menezes-Reis et al. ${ }^{(45)}$ found that Roussouly type 2 was associated with disc degeneration in the L4-L5 segment in asymptomatic individuals.

More recently, the first description of the sagittal alignment of the spine with degenerative disease based on its shape was published ${ }^{(46)}$, identifying 11 types. The newly proposed classification includes the four classic types of Roussouly along with seven other types found in degenerated spines: anteverted type 3; anteverted type 4; false type 2; false type 2 with thoracic kyphosis; false type 3, lumbar kyphosis; and global kyphosis. That study proposes a possible explanation for the fact that the classical subtypes seen in healthy individuals have evolved into pathological types.

Research in asymptomatic individuals has shown a correlation between spinopelvic parameters and paravertebral muscle volume, although none of those parameters have been found to correlate with fatty infiltration of mus$\mathrm{cle}^{(47)}$. The volume of the psoas muscle showed a positive correlation with the magnitude of the thoracic kyphosis, lumbar lordosis, and sagittal vertical axis ${ }^{(47)}$.

Previous studies have found that patients with lumbar pain have a decreased sacral slope, an increased pelvic tilt, and a reduced lumbar lordosis ${ }^{(37,48,49)}$. Barrey et al..$^{(37)}$ 
evaluated 57 patients with disc degeneration or herniation prior to arthrodesis, in comparison with 154 control subjects, and found the pelvic incidences to be similar between the two groups, although the former group showed lower values for sacral slope, lumbar lordosis, and thoracic kyphosis, as well as higher pelvic tilt values. Rajnics et al. ${ }^{(49)}$ also observed significant differences between patients with herniated disc-related lumbar pain $(n=50)$ and healthy individuals $(\mathrm{n}=30)$ in terms of the sacral slope, pelvic tilt, and lumbar lordosis parameters. The authors suggested that the lower sacral slope, higher pelvic tilt, and lower lumbar lordosis observed in the patients would lead to an increase in the compression forces applied to the anterior components (vertebral bodies and intervertebral discs), contributing to disc degeneration.

A recently introduced biplane X-ray imaging system (EOS; EOS Imaging, Paris, France) allows the simultaneous acquisition of full-body biplane projections (anteroposterior and lateral) with a radiation dose significantly lower than that of a single radiography, providing a complete evaluation of the spinal deformity and revealing any compensatory mechanisms recruited by the patient ${ }^{(46,50)}$. However, this technique is not yet widely available.

The measurement of multiple spinopelvic parameters offers a more complete view of the relationship between the deformity and its compensation. However, the assessment with multiple parameters can be complicated and requires time and experience. The use of a single parameter that combines anteversion of the trunk and the pelvic tilt tends to be a good option for the screening of possible deformities of the sagittal alignment. Future studies comparing the global tilt, spinopelvic angle, and $\mathrm{T} 1$ pelvic angle parameters should be encouraged.

\section{CONCLUSION}

We have presented a review of the spinopelvic parameters that are most relevant in clinical practice. A better understanding of the interaction between the spine and the pelvis, as well as of the compensatory mechanisms involved in the presence of deformities, allow the appropriate diagnosis and a more sophisticated approach to managing the care of patients with spinal deformities.

\section{REFERENCES}

1. Skoyles JR. Human balance, the evolution of bipedalism and dysequilibrium syndrome. Med Hypotheses. 2006;66:1060-8.

2. Le Huec JC, Saddiki R, Franke J, et al. Equilibrium of the human body and the gravity line: the basics. Eur Spine J. 2011;20 Suppl 5: 558-63.

3. Glassman SD, Bridwell K, Dimar JR, et al. The impact of positive sagittal balance in adult spinal deformity. Spine (Phila Pa 1976). 2005;30:2024-9.

4. Protopsaltis T, Schwab F, Bronsard N, et al. The T1 pelvic angle, a novel radiographic measure of global sagittal deformity, accounts for both spinal inclination and pelvic tilt and correlates with healthrelated quality of life. J Bone Joint Surg Am. 2014;96:1631-40.

5. Legaye J, Duval-Beaupère G, Hecquet J, et al. Pelvic incidence: a fundamental pelvic parameter for three-dimensional regulation of spinal sagittal curves. Eur Spine J. 1998;7:99-103.

6. Lafage V, Schwab F, Patel A, et al. Pelvic tilt and truncal inclination: two key radiographic parameters in the setting of adults with spinal deformity. Spine (Phila Pa 1976). 2009;34:E599-606.

7. Terran J, Schwab F, Shaffrey CI, et al. The SRS-Schwab adult spinal deformity classification: assessment and clinical correlations based on a prospective operative and nonoperative cohort. Neurosurgery. 2013;73:559-68.

8. Schwab F, Dubey A, Gamez L, et al. Adult scoliosis: prevalence, SF36 , and nutritional parameters in an elderly volunteer population. Spine (Phila Pa 1976). 2005;30:1082-5.

9. Barrey C, Roussouly P, Le Huec JC, et al. Compensatory mechanisms contributing to keep the sagittal balance of the spine. Eur Spine J. 2013;22 Suppl 6:S834-41.

10. During J, Goudfrooij H, Keessen W, et al. Toward standards for posture. Postural characteristics of the lower back system in normal and pathologic conditions. Spine (Phila Pa 1976). 1985;10:83-7.

11. Duval-Beaupère G, Schmidt C, Cosson P. A Barycentremetric study of the sagittal shape of spine and pelvis: the conditions required for an economic standing position. Ann Biomed Eng. 1992;20:451-62.

12. Le Huec JC, Faundez A, Dominguez D, et al. Evidence showing the relationship between sagittal balance and clinical outcomes in surgical treatment of degenerative spinal diseases: a literature review. Int Orthop. 2015;39:87-95.

13. Mehta VA, Amin A, Omeis I, et al. Implications of spinopelvic alignment for the spine surgeon. Neurosurgery. 2015;76 Suppl 1:S42-56.

14. Roussouly P, Gollogly S, Berthonnaud E, et al. Classification of the normal variation in the sagittal alignment of the human lumbar spine and pelvis in the standing position. Spine (Phila Pa 1976). 2005;30: 346-53.

15. Marks M, Stanford C, Newton P. Which lateral radiographic positioning technique provides the most reliable and functional representation of a patient's sagittal balance? Spine (Phila Pa 1976). 2009;34:949-54.

16. Van Royen BJ, Toussaint HM, Kingma I, et al. Accuracy of the sagittal vertical axis in a standing lateral radiograph as a measurement of balance in spinal deformities. Eur Spine J. 1998;7:408-12.

17. Schwab F, Patel A, Ungar B, et al. Adult spinal deformity-postoperative standing imbalance: how much can you tolerate? An overview of key parameters in assessing alignment and planning corrective surgery. Spine (Phila Pa 1976). 2010;35:2224-31.

18. Jackson RP, Kanemura T, Kawakami N, et al. Lumbopelvic lordosis and pelvic balance on repeated standing lateral radiographs of adult volunteers and untreated patients with constant low back pain. Spine (Phila Pa 1976). 2000;25:575-86.

19. Roussouly P, Gollogly S, Noseda O, et al. The vertical projection of the sum of the ground reactive forces of a standing patient is not the same as the $\mathrm{C} 7$ plumb line: a radiographic study of the sagittal alignment of 153 asymptomatic volunteers. Spine (Phila Pa 1976). 2006;31:E320-5.

20. Kobayashi T, Atsuta Y, Matsuno T, et al. A longitudinal study of congruent sagittal spinal alignment in an adult cohort. Spine (Phila Pa 1976). 2004;29:671-6.

21. Roussouly P, Pinheiro-Franco JL. Biomechanical analysis of the spino-pelvic organization and adaptation in pathology. Eur Spine J. 2011;20 Suppl 5:609-18.

22. Schwab F, Lafage V, Patel A, et al. Sagittal plane considerations and the pelvis in the adult patient. Spine (Phila Pa 1976). 2009;34: 1828-33.

23. Bernhardt M, Bridwell KH. Segmental analysis of the sagittal plane alignment of the normal thoracic and lumbar spines and thoracolumbar junction. Spine (Phila Pa 1976). 1989;14:717-21.

24. Iyer S, Sheha E, Fu MC, et al. Sagittal spinal alignment in adult spinal deformity: an overview of current concepts and a critical analysis review. JBJS Rev. 2018;6:e2. 
25. Lafage V, Schwab F, Skalli W, et al. Standing balance and sagittal plane spinal deformity: analysis of spinopelvic and gravity line parameters. Spine (Phila Pa 1976). 2008;33:1572-8.

26. Schwab F, Lafage V, Boyce R, et al. Gravity line analysis in adult volunteers: age-related correlation with spinal parameters, pelvic parameters, and foot position. Spine (Phila Pa 1976). 2006;31:E959-67.

27. Legaye J, Duval-Beaupère G. Sagittal plane alignment of the spine and gravity: a radiological and clinical evaluation. Acta Orthop Belg. 2005; 71:213-20.

28. Schwab FJ, Blondel B, Bess S, et al. Radiographical spinopelvic parameters and disability in the setting of adult spinal deformity: a prospective multicenter analysis. Spine (Phila Pa 1976). 2013;38:E803-12.

29. Lazennec JY, Ramaré S, Arafati N, et al. Sagittal alignment in lumbosacral fusion: relations between radiological parameters and pain. Eur Spine J. 2000;9:47-55.

30. Obeid I, Boissière L, Yilgor C, et al. Global tilt: a single parameter incorporating spinal and pelvic sagittal parameters and least affected by patient positioning. Eur Spine J. 2016;25:3644-9.

31. Banno T, Togawa D, Arima H, et al. The cohort study for the determination of reference values for spinopelvic parameters ( $\mathrm{T} 1$ pelvic angle and global tilt) in elderly volunteers. Eur Spine J. 2016; 25:3687-93.

32. Mac-Thiong JM, Roussouly P, Berthonnaud E, et al. Sagittal parameters of global spinal balance: normative values from a prospective cohort of seven hundred nine Caucasian asymptomatic adults. Spine (Phila Pa 1976). 2010;35:E1 193-8.

33. Son SM, Shin JK, Goh TS, et al. Predictive findings of the presence of stooping in patients with lumbar degenerative kyphosis by upright whole spine lateral radiography. Spine (Phila Pa 1976). 2018;43:571-7.

34. Lee HS, Lee JS, Shin JK, et al. Correlations between sagittal spinal balance and quality of life in rheumatoid arthritis. Clin Spine Surg. 2017;30:E412-E417.

35. Pellet N, Aunoble S, Meyrat R, et al. Sagittal balance parameters influence indications for lumbar disc arthroplasty or ALIF. Eur Spine J. 2011;20 Suppl 5:647-62.

36. Morvan G, Mathieu P, Vuillemin V, et al. Standardized way for imaging of the sagittal spinal balance. Eur Spine J. 2011;20 Suppl 5:602-8.

37. Barrey C, Jund J, Noseda O, et al. Sagittal balance of the pelvisspine complex and lumbar degenerative diseases. A comparative study about 85 cases. Eur Spine J. 2007;16:1459-67.
38. Obeid I, Hauger O, Aunoble S, et al. Global analysis of sagittal spinal alignment in major deformities: correlation between lack of lumbar lordosis and flexion of the knee. Eur Spine J. 201 1;20 Suppl 5:681-5.

39. Barrey C, Roussouly P, Perrin G, et al. Sagittal balance disorders in severe degenerative spine. Can we identify the compensatory mechanisms? Eur Spine J. 2011 ; 20 Suppl 5:626-33.

40. Roussouly P, Nnadi C. Sagittal plane deformity: an overview of interpretation and management. Eur Spine J. 2010;19:1824-36.

41. Potter BK, Lenke LG, Kuklo TR. Prevention and management of iatrogenic flatback deformity. J Bone Joint Surg Am. 2004;86: 1793 808.

42. Mardare M, Oprea M, Popa I, et al. Sagittal balance parameters correlate with spinal conformational type and MRI changes in lumbar degenerative disc disease: results of a retrospective study. Eur J Orthop Surg Traumatol. 2016;26:735-43.

43. Lazennec JY, Riwan A, Gravez F, et al. Hip spine relationships: application to total hip arthroplasty. Hip Int. 2007;17 Suppl 5:S91-104.

44. Chaléat-Valayer E, Mac-Thiong JM, Paquet J, et al. Sagittal spinopelvic alignment in chronic low back pain. Eur Spine J. 2011;20 Suppl 5:634-40.

45. Menezes-Reis R, Bonugli GP, Dalto VF, et al. Association between lumbar spine sagittal alignment and L4-L5 disc degeneration among asymptomatic young adults. Spine (Phila Pa 1976). 2016;41: E1081-7.

46. Sebaaly A, Grobost P, Mallam L, et al. Description of the sagittal alignment of the degenerative human spine. Eur Spine J. 2018; 27:489-96.

47. Menezes-Reis R, Bonugli GP, Salmon CEG, et al. Relationship of spinal alignment with muscular volume and fat infiltration of lumbar trunk muscles. PLoS One. 2018;13:e0200198.

48. Jackson RP, McManus AC. Radiographic analysis of sagittal plane alignment and balance in standing volunteers and patients with low back pain matched for age, sex, and size. A prospective controlled clinical study. Spine (Phila Pa 1976). 1994;19:1611-8.

49. Rajnics $P$, Templier A, Skalli W, et al. The importance of spinopelvic parameters in patients with lumbar disc lesions. Int Orthop. 2002; 26:104-8.

50. Dubousset J, Charpak G, Dorion I, et al. A new 2D and 3D imaging approach to musculoskeletal physiology and pathology with low-dose radiation and the standing position: the EOS system. Bull Acad Natl Med. 2005;189:287-300. 